\title{
Planning the Size and Organization of KLM's Aircraft Maintenance Personnel
}

\author{
MatThijs C. DiJKSTRA \\ LEO G. KROON \\ MARC SALOMON \\ Alpha Ciboma, Polanerbaan 13a NL-3447 GN \\ Woerden, The Netherlands \\ Rotterdam School of Management \\ Erasmus University, PO Box 1738 \\ NL-3000 DR Rotterdam, The Netherlands \\ Rotterdam School of Management \\ and ORTEC Consultants bo \\ Groningenweg 6-02 \\ NL-2803 PV Gouda, The Netherlands \\ Jo A. E. E. VAN NUNEN \\ Rotterdam School of Management \\ Erasmus University \\ LuK N. VAN WASSENHOVE \\ INSEAD, Boulevard de Constance \\ F-77305 Fontainebleau Cedex, France
}

We developed a decision support system (DSS) for the aircraft maintenance department of KLM Royal Dutch Airlines at Schiphol Airport. This department inspects and maintains aircraft during their ground time at the airport. Its main resource is its workforce. Since January 1990 the DSS has supported management in analyzing several capacity planning problems related to the size and the organization of the workforce. In particular, management uses the DSS to determine the appropriate number of maintenance engineers and their training requirements, and to analyze the efficiency and effectiveness of the maintenance department.

$\mathrm{K}$

LM Royal Dutch Airlines has been the major Dutch carrier since 1919. KLM's home base is Schiphol Airport near Amsterdam. Currently (1993), KLM owns about 90 aircraft of eight different types. With this fleet, KLM operates flights to about 150 cities in 79 countries. Of course, the safety of passengers and crew has top priority. To guarantee safety, KLM carries out high quality aircraft maintenance, rely- ing on about 3,000 employees in its maintenance department. They also carry out maintenance operations on aircraft belonging to about 30 other carriers that have maintenance contracts with KLM.

Preventive aircraft maintenance consists partly of major inspections and partly of minor inspections. Major inspections are performed in KLM's hangars after a certain number of flight hours, depending on the 
aircraft type. Major inspections take from several hours to several months. The longest major inspections may involve checking all the individual parts of an aircraft. Minor inspections are conducted during the ground time between arrival and departure at the airport. A minor inspection, also called a project, includes the following services:

-Arrival services, which consist of fixing ground power supply, compiling a list of technical complaints based on the crew's flight records, and collecting resources (such as mobile cranes and scaffoldings) for the platform services,

-Platform services, which consist of checking the technical state of the aircraft and, if necessary, performing repairs, and -Departure services, which consist of performing a final technical check of the aircraft.

Maintenance engineers from KLM's aircraft maintenance group VOC (Vliegtuig Onderhoud Centrum in Dutch) carry out these tasks. The workload of KLM-VOC's technical staff is mainly based on

-KLM's timetables and those of other carriers,

-Contracts with other carriers, and

-Maintenance standards.

The maintenance standards specify (1) in which time interval KLM must schedule each service, (2) how much time must be spent on each service, and (3) the skills required for each service (there exist mechanical-, electrical-, or radio-skilled engineers). Usually, maintenance standards are specified by aircraft manufacturers, governments, and the carriers themselves. KLM's timetable and those of other carriers usually have cyclical patterns, with a cycle length of one week. As a result, the workload of KLM-VOC also shows a cyclical pattern. Furthermore, the workload on an average day shows some clearly distinguishable peaks, caused by KLM's desire to limit the waiting times for transit passengers. For example, early in the morning a stream of intercontinental flights arrives at Schiphol Airport. Shortly thereafter a stream of continental flights departs from Schiphol Airport to several destinations in Europe.

KLM wants to increase the utilization rate of its fleet and to smooth the workload of its ground service departments. It can accomplish the latter by increasing the number of peaks and reducing the size of the peaks. KLM-VOC's management is interested both in aggregated and in more detailed information on the workload pattern, since this provides valuable insights

\section{KLM wants to smooth the workload of its ground service departments.}

into the size and organization of the workforce it needs. Figure 1 shows a typical workload pattern.

KLM-VOC's workforce consists of about 250 ground engineers and 150 nontechnical employees. The ground engineers are highly skilled and well-trained employees, since their job is a very responsible one. A governmental rule specifies that an engineer is allowed to carry out inspections on a specific aircraft type only if licensed for that aircraft type. Besides a license for an aircraft type, an engineer also has a specific skill for mechanical, electrical, or radio 

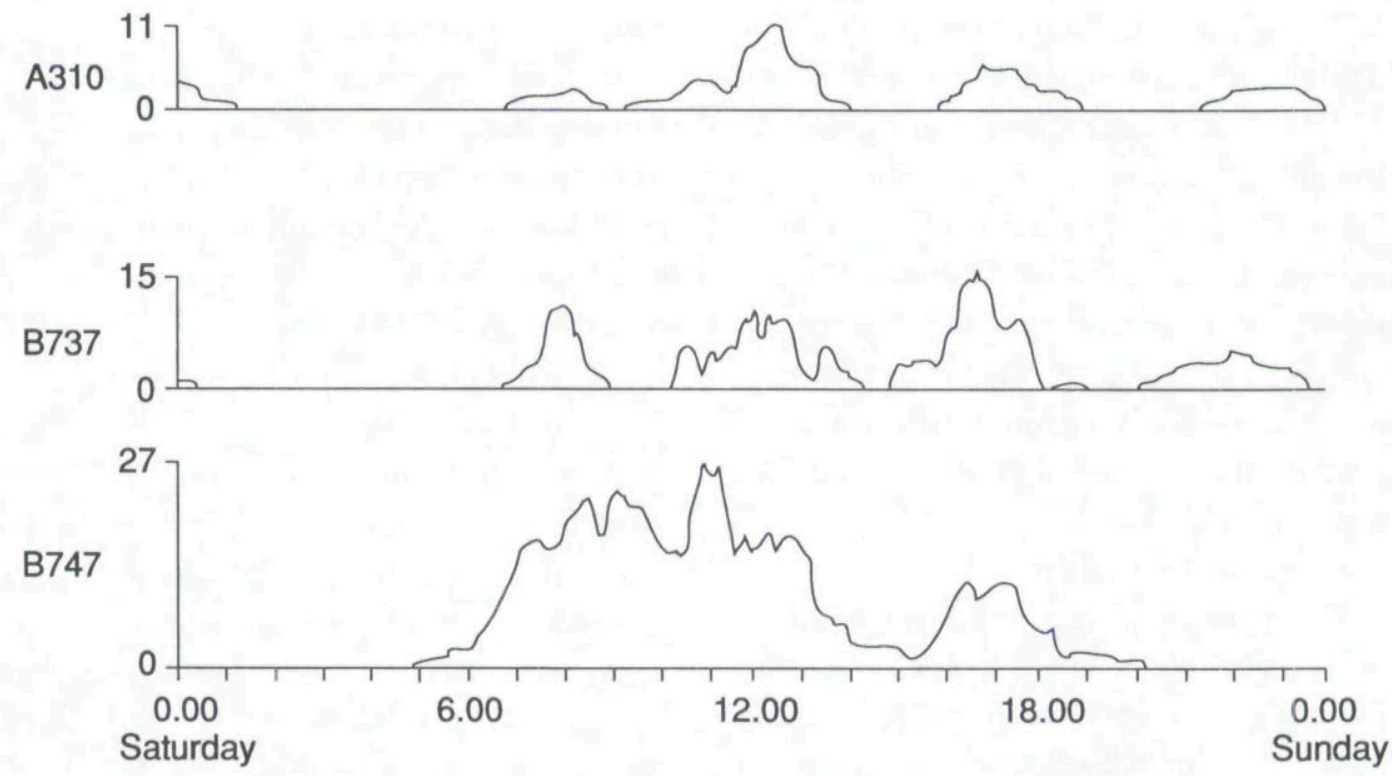

Figure 1: The workload between Saturday midnight and Sunday midnight for mechanical engineers contains at most 11 parallel jobs on A310s, 15 parallel jobs on B737s, and 27 parallel jobs on B747s.

operations. The engineers obtain their licenses for aircraft types and skills by attending training programs consisting of theoretical and practical courses, field training, and exams. Depending on the engineer's experience, it takes several months to several years to complete a training program. In addition to inspections that require licensed and skilled employees, KLM-VOC carries out a small number of jobs for which no license is required.

It would be preferable if all engineers had licenses for all aircraft types and all skills. Then, they would be totally flexible. However, KLM's internal safety rules limit engineers to licenses for at most two aircraft types and one skill.

The engineers operate in teams, which are KLM-VOC's smallest organizational subunits. Currently there are 12 teams of about 20 engineers each. KLM assigns en- gineers to teams so that the teams are almost identical with respect to their available licenses and skills (Table 1).

The teams operate in a four-shift system with an early day shift from 6:00 AM to

\section{Skills}

Licenses Mechanical Electrical Radio

$\begin{array}{llll}\text { A310/B747 } & 2 & & 1 \\ \text { B737/B747 } & 1 & & 1 \\ \text { B737/DC10 } & 1 & 1 & 1 \\ \text { A310/B737 } & 1 & 1 & \\ \text { A310/DC9 } & 1 & 1 & 1 \\ \text { A310 } & 1 & 1 & \\ \text { B747 } & 3 & 1 & 1\end{array}$

Table 1: Each maintenance team has 20 engineers, each having a specific license-skill combination. For example, two engineers in the team have obtained a mechanical skill with licenses for A310 and B747. 
3:00 PM, a late day shift from 10:00 AM to 6:00 PM, an evening shift from 3:00 PM to 11:00 PM, and a night shift from 11:00 PM to 6:00 AM (Figure 2). The assignment of teams to shifts is constrained by several governmental, union, and internal KLM rules. For instance, the average number of shifts per week for each team should be five, and each team should have at least one day off between a night shift and the next day shift.

\section{Managerial Problems}

The managers' main problem is to find a good match between workload and workforce. The elements that play a role in this match are time tables, maintenance norms, and contracts for the workload, shifts, and teams of the workforce (Figure 3).

The quality of the match in a certain planning period (day, week, or month) is expressed in terms of the service level and the utilization rate. These performance indicators are defined as follows:

service level

$$
\begin{aligned}
\text { \# of maintenance jobs } \\
\text { carried out in time } \\
\text { total \# of maintenance jobs }
\end{aligned}
$$

utilization rate

$$
=\frac{\text { total \# of productive man hours }}{\text { total \# of available man hours }} \text {. }
$$

The main consequences of a bad match are -A low service level, caused by too high utilization of the workforce. A low service level corresponds to delays, which should

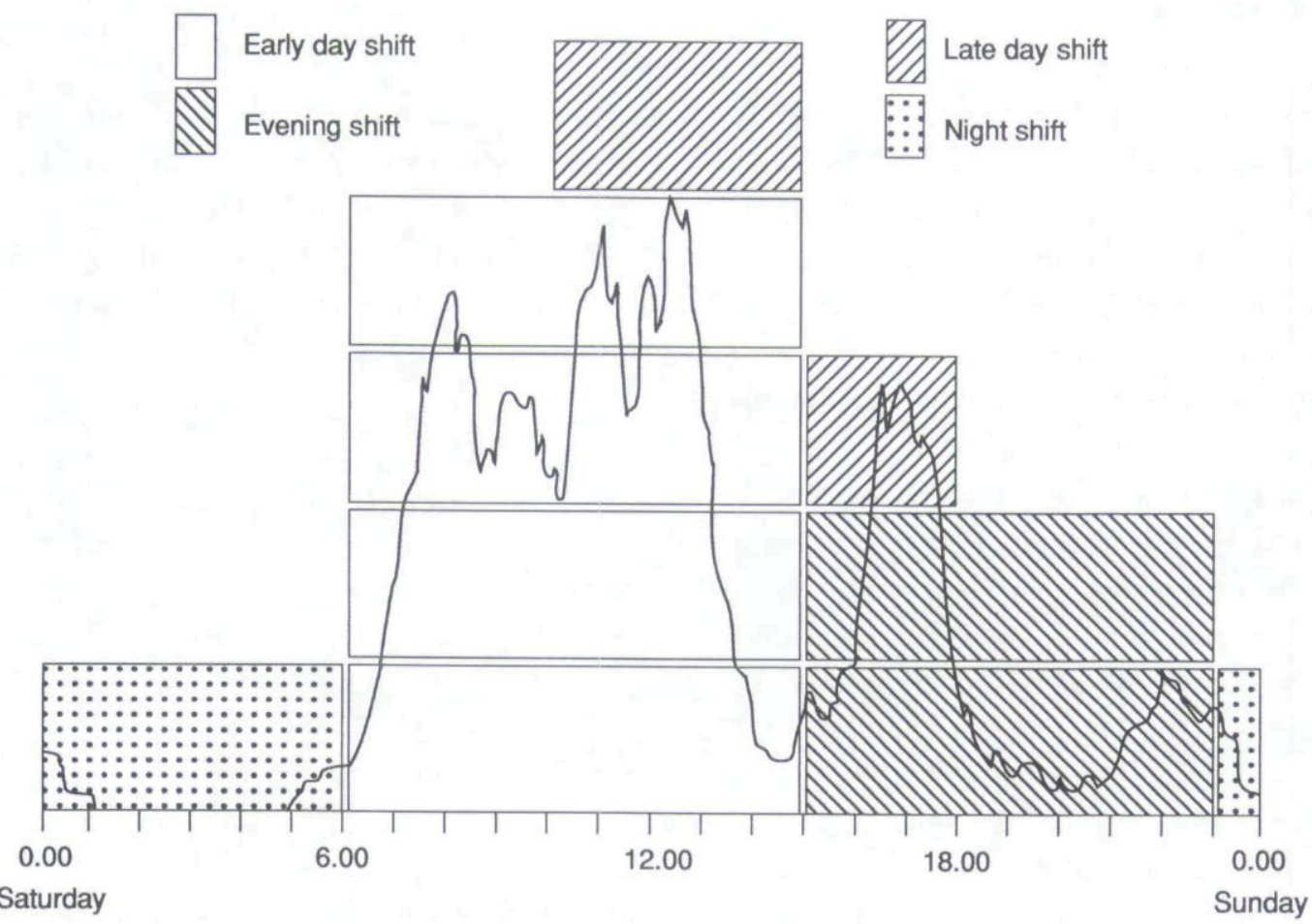

Figure 2: In this scheme for a typical Saturday, each team of engineers is represented by a block. For example, four teams of engineers are available in the early day shift (6:00 AM-3:00 PM). 


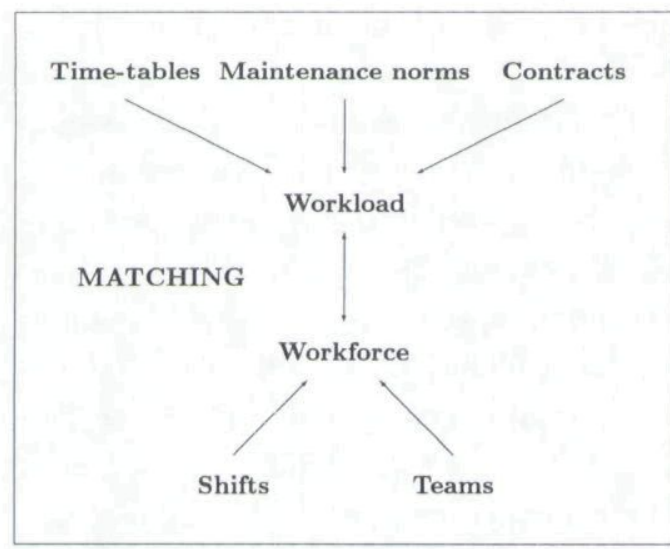

Figure 3: Various components contribute to the problem of matching the workforce to the workload.

be avoided because they cause customer dissatisfaction and high costs,

-A low utilization rate of the workforce, caused by too many engineers, or engineers with inappropriate licenses or skills being assigned to a shift. Of course, both lead to an inefficient and hence costly maintenance organization.

Planning for a good match of workload and workforce is therefore important, and it involves both strategic and tactical planning. At the strategic level, KLM-VOC's management must

- Set an appropriate target for the service level,

-Obtain insight into the relationship between the size and organization of the workforce and the resulting service level, -Determine the impact of timetable adjustments on the size and organization of the workforce,

-Analyze the consequences of the introduction of new aircraft types on the size and organization of the workforce, and -Evaluate the consequences of partnerships with other companies with respect to maintenance.

KLM-VOC's management is also faced with a number of problems at the tactical decision level. Examples are

-To evaluate the financial implications of potential new contracts with other carriers, -To determine the number of shifts per day and the beginning and ending times of each shift,

-To compose the teams appropriately with respect to license combinations and skills,

- To develop a training program, that is, to determine the capacity and contents of the educational program needed for maintenance engineers, and -To estimate next year's personnel budgets for the maintenance department.

The management of the maintenance department uses the DSS in dealing with these problems. It focuses in particular on the problems of coping with different aircraft types, license combinations, and skills. Before we developed the DSS, the managers based their strategic and tactical capacity planning on rough aggregate workload calculations for the various aircraft types. They could not evaluate the problems caused by the different aircraft types, license combinations, and skills to a sufficient level of detail. Furthermore, carrying out these calculations was very timeconsuming. KLM-VOC's management had the impression that the quality of decision making could be improved by the introduction of a DSS.

\section{Decision Support System}

The DSS consists of a database module, an analysis module, and a graphical user interface. The database module stores generic data, data about the workload, and 
data about the workforce. The generic data include information on carriers for which KLM-VOC carries out maintenance operations, and information on aircraft types. Data on the workload consist of arrival and departure times of aircraft at Schiphol and maintenance standards. Arrival and departure times are taken directly from the timetables of the airline companies. The maintenance standards specify (1) the total time required for each maintenance operation, (2) the allowable time interval during which a maintenance operation must be carried out relative to a plane's arrival and departure time (for example, a specific maintenance operation should not start earlier than 10 minutes after arrival and should not be finished later than 20 minutes before departure), and (3) that maintenance operations should start as early as possible. Finally, data on the workforce consist of the license-skill combinations within a team, and the shift schedules that specify the beginning and ending times of the shifts, as well as the number of teams per shift.

A complete set of tables with generic data, data on the workload, and data on the workforce is called a scenario. The database module provides functions for operations on complete scenarios and functions for operations on individual tables of a selected scenario. By analyzing different scenarios the user of the DSS obtains valuable insight into the effects of changes in the data.

The analysis module provides extensive possibilities for analyzing scenarios. It consists of routines for (1) estimating the workload, (2) optimizing the size and organization of the workforce, and (3) evaluat- ing the quality of the match between workload and workforce.

The routine for estimating the workload is based on the maintenance operations and the maintenance standards. For example, consider mechanical work related to a platform inspection of a Boeing B747 on incoming flight KL-342 with arrival time 9:00 AM and departure time 11:25 AM. Total ground time at Schiphol is 145 minutes. The maintenance standard for the mechan-

\section{The actual workload may differ from the estimated workload.}

ical part of the platform inspection of a Boeing B747 specifies that (1) the total time required to carry out the work is $160 \mathrm{~min}$ utes, (2) the operations should take place in the interval from 15 minutes after arrival to 30 minutes before departure, and (3) the operations should start as early as possible. Since only 100 minutes are available for the platform inspection, the operations are carried out by two engineers with 80 minutes of work for each. Both engineers will be scheduled to start their work 15 minutes after arrival (9:15 AM), and will be finished 80 minutes later (10:35 AM).

All maintenance operations carried out by KLM-VOC are translated into estimated workloads by calculations like these. Figure 1 shows an example of a resulting workload pattern. Due to circumstances (delays, failures), the actual workload may differ from the estimated workload. The influences of such irregularities on operations can be analyzed by simulating these circumstances within a scenario (for exam- 
ple, changing maintenance standards or generating delays on arrival times).

The routine for optimizing the size and organization of the workforce uses the following input:

-Workload estimates per aircraft type (output of the workload estimation routine) in terms of jobs with fixed starting times, fixed finishing times, and required skill,

- Beginning and ending times of the shifts,

- The number of teams per shift, and

- The required service level.

Based on this input, the optimization routine calculates the number of engineers per team with a particular license-skill combination and assigns engineers to maintenance jobs so that (1) the total number of engineers is minimal, (2) the service level constraint is satisfied, and (3) each job is carried out by an engineer with an appropriate license-skill combination. For example, if the service level is set to 1.00 , the system computes the number of engineers per team with a particular licenseskill combination, such that all maintenance jobs are carried out and the total number of engineers is minimal. In case of a user-specified service level of 0.98 , the system calculates a composition of the teams such that at least 98 percent of the maintenance jobs are carried out. We describe a simplified version of the integer programming model used by the optimization routine in the appendix (model 1). A heuristic procedure within the optimization routine quickly generates good solutions to this model.

The routine for evaluating the maintenance processes within KLM-VOC deter- mines the quality of the match between workload and workforce. It measures the quality of the match in terms of service level and utilization rate. The evaluation routine uses the following input:

-Workload estimates per aircraft type (output of the workload estimation routine) in terms of jobs with fixed starting times, fixed finishing times, and required skill,

- Beginning and ending times of the shifts,

- The number of teams per shift, and

- The number of engineers per team and their license-skill combinations.

Based on the input, the evaluation routine assigns engineers to maintenance jobs so that the service level is maximized. The results of the procedure can be presented graphically (Figures 4 and 5). We describe the model underlying the evaluation routine in the appendix (model 2). Within the system, it is solved by a fast and effective heuristic procedure.

The optimization routine and the evaluation routine differ from each other in the

\section{The managers consider it a valuable tool for analyzing strategic and tactical problems.}

following sense: the optimization routine considers the service level as given and determines the best composition of the teams with respect to the number of engineers required and their license-skill combinations, whereas the evaluation routine considers the composition of the teams as given and determines the maximum number of maintenance jobs that can be carried 
$\square$ Fulfilled Unfulfilled
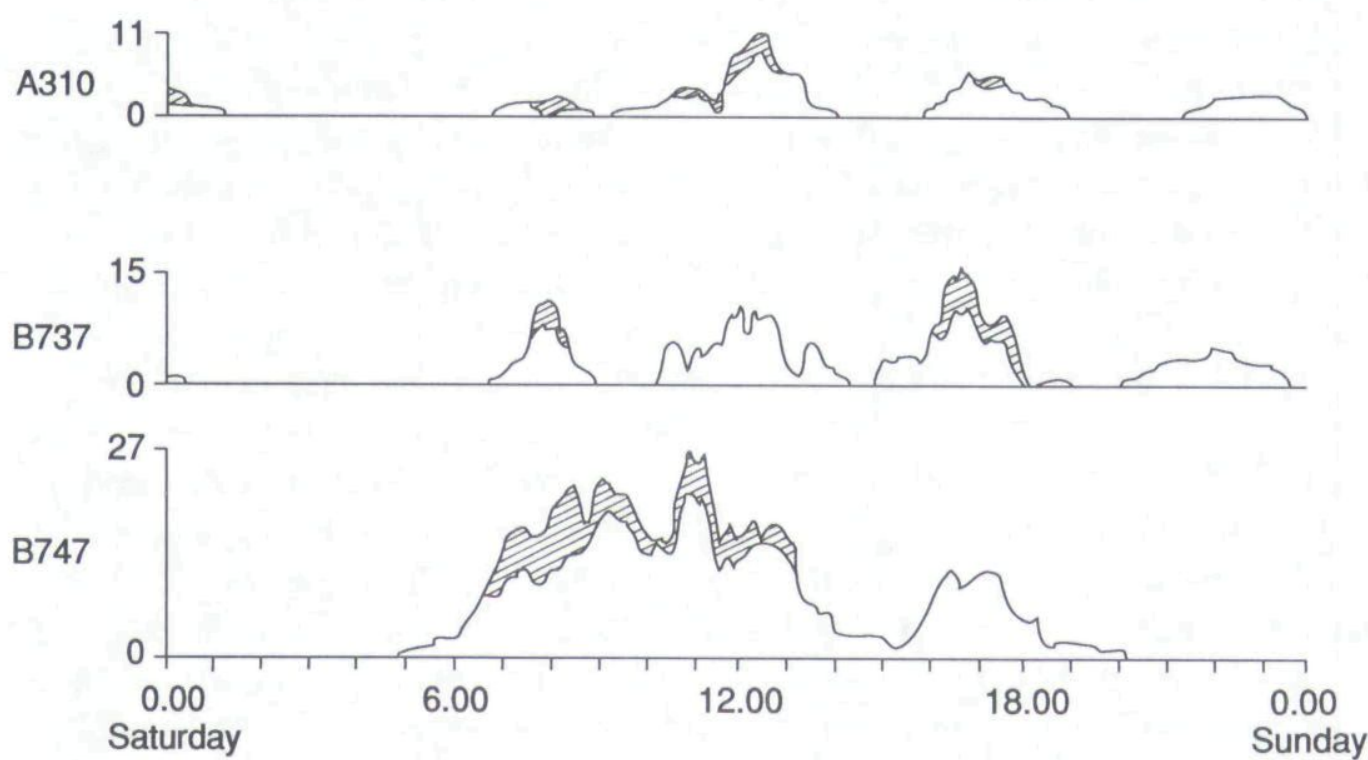

Figure 4: Part of the mechanical work needed on a typical Saturday for aircraft types A310, B737, and B747 can be carried out (fulfilled) by the available engineers (white area), and part of the work (unfulfilled) cannot be done in time (shaded area).

out in time (that is, it maximizes the service level).

Since it is not feasible to change the composition of the teams in the short run (the educational program to obtain new licenses takes several months to several years) and since in the long run management wants to design the composition of the teams so that in principle all maintenance work can be carried out (service level 1.00), KLM uses the optimization routine mainly in strategic studies and the evaluation routine in tactical studies.

\section{System Development and Usage}

In 1988, KLM set up a project to develop the DSS. The project team consisted of five persons from academia and five persons from KLM-VOC. At the start of the project, management had only a rough idea of the problems the DSS should handle and of the look and feel of the system. To structure the decision problems and to determine the functional requirements of the system, we adopted a prototyping approach. One functional requirement management set was that the DSS should be stand-alone and should interface with the database systems on KLM's mainframe. Another functional requirement was that the system should run on personal computers on the employees' desks.

We developed the database module of the system using a tool for generating databases based on Clipper, and we developed the analysis module and the graphical user interface using Borland's Turbo Pascal and some tools for graphics. We devoted a great deal of effort to making the 

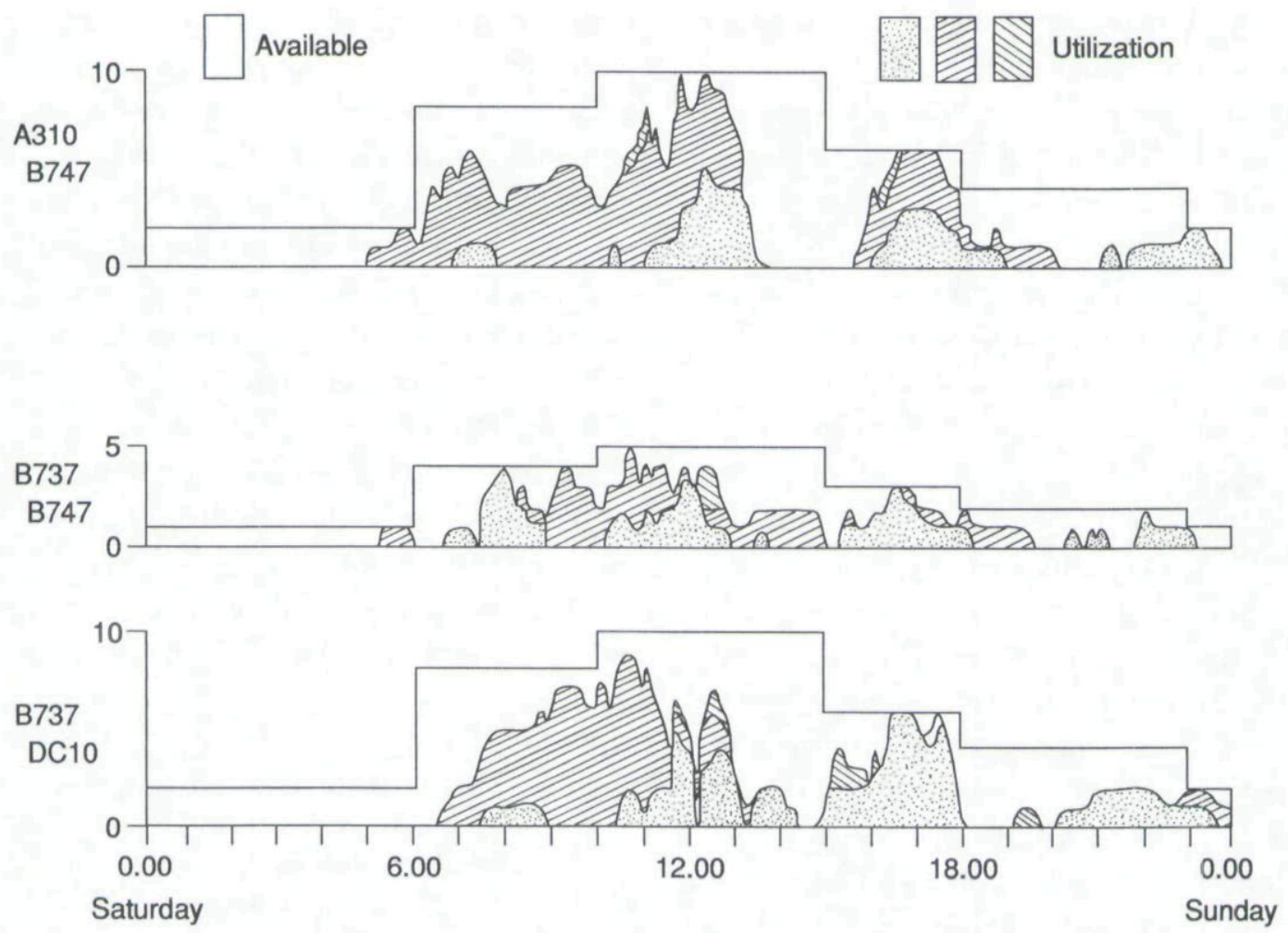

Figure 5: The assignment of mechanically skilled engineers to maintenance jobs on a typical Saturday for license combinations A310/B747, B737/B747, and B737/DC10. The white area indicates how many engineers are available (at midnight, one for B737/B747, two for A310/B747, and two for B737/DC10, growing to five for B737/B747, 10 for A310/B747, and 10 for B737/ $\mathrm{DC} 10$ around midday). The other areas reflect how many of these engineers are assigned to each aircraft type: the dotted area for the engineers' first license, and the striped area (with inclining pattern) for the engineers' second license. The striped area (with declining pattern) indicates work for which no license is required. For example, for engineers with license combination A310/B747, the dotted area shows how many engineers work on A310s (first license), and the inclining stripes shows how many engineers work on B747s (second license).

system user friendly, which resulted in quick user acceptance. We installed the system in 1990, and KLM-VOC has used it since then.

The DSS is used mainly by staff employees to answer questions posed by KLM-

VOC's managers. The system has provided valuable support in the following studies:

-A study on the impact of new time-table structures (for example, time-tables with an increased number of arrival and depar- ture peaks per day),

- A study on the effects of allowing engineers to hold licenses for three aircraft types instead of two, -A study to estimate the workforce required per group of gates, in case maintenance processes become decentralized per gate group at some point in the future, -A study to determine the number of engineers and their license-skill combinations required for 1992 and 1993, and 
-A study on the effects of contracts with other carriers.

The DSS provides KLM-VOC's management with information that was either not available before or was too time-consuming to collect within a short planning cycle. It increases their insight into the various problems that must be solved within the maintenance department. The managers consider it a valuable tool for analyzing strategic and tactical problems. The users of the system even advocated its use to other departments of KLM, such as the helicopter department.

However, evaluating a DSS is generally more difficult than evaluating more traditional information systems, since simple criteria like costs and benefits are hardly useful [Keen 1981]. First, a DSS is never completely finished and therefore costs are difficult to specify. Second, the benefits of a DSS are often largely qualitative, for example, the impact on the organization, the quality of decision-making processes, and the resulting decisions. Evaluating these benefits in quantitative terms is difficult and has not yet received much attention in the literature [Elam, Huber, and Hurt 1986].

When considering the results of a DSS, it is important to keep in mind that they are based on mathematical models that are abstractions of reality. Optimality in mathematical terms need not necessarily match optimality in practical terms. Furthermore, most of the calculations within a DSS are based on approximation algorithms. Therefore, the results of a DSS must be handled with care. The user of the DSS must judge the practical value of a solution in light of qualitative or quantitative considerations that were not explicitly taken into account by the models within the DSS. Hence, a DSS must be used in an interactive way, where the intelligence of the user is combined with the capability of the DSS to organize and process enormous amounts of data and to solve complex mathematical decision problems using sophisticated operational research techniques.

\section{Acknowledgements}

We are grateful to René Kalmann, Dolf Beltz, Paul Chün, Thom Grobben, and Jan Smit of KLM's maintenance department for their cooperation and helpful comments during all development phases of the DSS.

\section{APPENDIX}

We present a mathematical description of the problems solved within the analysis module. Recall the following assumptions: (A1) the number of teams is fixed, (A2) all teams have the same number of engineers with a particular license-skill combination, and (A3) each engineer has exactly one skill,

To keep the presentation clear, we first describe the situation in which the service level equals 1.00 and in which there is only one shift and one skill. However, as Kroon [1990] and the model extensions below show, it is easy to modify the model in such a way that (1) lower service levels, (2) multiple shifts, and (3) multiple skills are properly taken into account.

We suppose that the set $J$ of jobs (services) has to be carried out. Job $j \in J$ requires continuous processing in the interval $\left(s_{j}, f_{j}\right)$ and is related to an aircraft of type $a_{j}$. Each engineer is assumed to have a license combination that specifies the aircraft types he is allowed to work on. The set of different license combinations is denoted by $C$. Furthermore, the set $J_{c}$ denotes the set of jobs that can be carried out by engineers with license combination $c$. Con- 
versely, we use the notation $C_{i}$ for the set of license combinations that can be used for carrying out job $j \in J$. Furthermore, $\left\{t_{p} \mid p \in P\right\}$ is the set of starting times of the jobs. That is $\left\{t_{p} \mid p \in P\right\}=\left\{s_{j} \mid j \in J\right\}$.

\section{Model 1 (Optimization Routine)}

Here the problem is to determine the minimum number of engineers with appropriate license combinations, such that all jobs can be carried out. Given the assumptions A1 and A2, minimizing the total number of engineers is equivalent to minimizing the number of engineers per team. The decision variables of the related integer program are defined as follows: $X_{j c}=\mathrm{a}$ binary variable indicating whether job $j$ has to be carried out by an engineer with license combination $c \in C_{j}$ and

$Y_{c}=$ an integer variable indicating the number of engineers with license combination $c$ in each team.

In terms of these decision variables, the objective and the constraints of Model 1 are

$\min Q=\sum_{c \in C} Y_{c}$

subject to

$$
\begin{aligned}
& \sum_{c \in C_{j}} X_{j c}=1 \text { for } j \in J \\
& \qquad \quad X_{k} \leq Y_{c} \text { for } c \in C, \quad p \in P
\end{aligned}
$$

all variables are integer.

The objective function (1) expresses that we are interested in minimizing the number of engineers per team. The constraints (2) guarantee that each job is carried out exactly once. The constraints (3) specify that the maximum job overlap of the jobs that are assigned to the engineers with license combination $c$ should not exceed the number of available engineers with license combination $c$. This implies that a feasible solution to the integer program can be transformed into a feasible assignment of jobs to engineers and vice versa. Finally, the integrality constraints (4) specify the integer character of the decision variables.

Kolen and Kroon [1992] show that in general this optimization problem belongs to the class of NP-hard problems. Kroon [1990] and Kroon, Salomon, and Van Wassenhove [1993] present algorithms that can be used to find optimal or approximate solutions.

\section{Model 2 (Evaluation Routine)}

Here the problem is to determine the maximum number of jobs that can be carried out (that is, maximum service level) given the size and the composition of the workforce. The prespecified number of engineers with license combination $c$ is known and denoted by $M_{c}$. In terms of the decision variables $X_{j c}$ (see Model 1 ), the objective and the constraints of Model 2 are stated as follows:

$\max Q=\sum_{j \in I} \sum_{c \in C_{j}} X_{j}$

subject to

$\sum_{c \in C_{j}} X_{j c} \leq 1$ for $j \in J$

$\sum_{\left\{;\left.\in\right|_{c} \mid s, \leq t_{p}<f_{j}\right\}} X_{j c} \leq M_{c} \quad$ for $c \in C, \quad p \in P$

all variables are integer.

(3) The objective function (5) expresses that we are interested in maximizing the number of jobs that are carried out. The constraints (6) guarantee that each job is carried out at most once. The interpretation of the constraints (7) and (8) is similar to that of the constraints (3) and (4) in Model 1.

Kolen and Kroon [1991] show that in general this optimization problem belongs to the class of NP-hard problems. Algorithms that can be used to find optimal or approximate solutions are described by Kroon, Salomon, and Van Wassenhove [1992].

\section{Model Extensions}

We now briefly discuss three extensions 
of Model 1 corresponding to (1) service levels less than 1.00, (2) multiple shifts, and (3) multiple skills.

(1) Service level less than 1.00: In this case constraint (2) is replaced by constraints $\left(2^{\prime}\right)$ and $\left(2^{\prime \prime}\right)$.

$$
\begin{aligned}
& \sum_{c \in C_{j}} X_{j c} \leq 1 \quad \text { for } \quad j \in J \\
& \sum_{j \in I} \sum_{c \in C_{j}} X_{j c} \geq \alpha|J|
\end{aligned}
$$

where $\alpha(0 \leq \alpha \leq 1)$ is the required service level.

(2) Multiple shifts: In order to take into account multiple shifts, constraint (3) is replaced by constraint ( $\left.3^{\prime}\right)$.

$$
\sum_{\left\{,\left|\epsilon_{c}\right| s, s \leq t_{p}<f_{i}:\right.} X_{j c} \leq N_{p} Y_{c} \text { for } c \in C, p \in P
$$

where $N_{p}$ is the number of teams present in the time interval $\left(t_{p}, t_{p+1}\right)$. The numbers $N_{p}$ are user-input and follow from the beginning and ending times of the shifts and from the number of teams per shift. Note that $\left(3^{\prime}\right)$ is based on assumption A2, which states that teams have identical compositions with respect to licenses.

(3) Multiple skills: Since each engineer has one skill only (assumption A3), the multiple-skill problem is solved by decomposing it into several one-skill problems as formulated by Model 1 .

Note that Model 2 can be extended to include multiple shifts and multiple skills analogous to Model 1.

\section{References}

Elam, J. J.; Huber, G. P.; and Hurt, M. E. 1986, "An examination of DSS literature (19751985)," in Decision Support Systems: A Decade in Perspective, eds. E. R. McLean and H. G. Sol, North Holland, Amsterdam, The Netherlands.

Keen, P. G. W. 1981, "Value analysis: Justifying decision support systems," MIS Quarterly, Vol. 5 (March), pp. 1-15.
Kolen, A. W. J. and Kroon, L. G. 1991, "On the computational complexity of (maximum) class scheduling," European Journal of Operational Research, Vol. 54, No. 1, pp. 23-38.

Kolen, A. W. J. and Kroon, L. G. 1992, "License class design: Complexity and algorithms," European Journal of Operational Research, Vol. 63, No. 3, pp. 432-444.

Kroon, L. G. 1990, "Job scheduling and capacity planning in aircraft maintenance," $\mathrm{PhD}$ diss., Erasmus University, Rotterdam, The Netherlands.

Kroon, L. G.; Salomon, M.; and Van Wassenhove, L. N. 1992, "Exact and approximation algorithms for the operational fixed interval scheduling problem," Management Report Series 103, Erasmus University, Rotterdam, The Netherlands. (Forthcoming in European Journal of Operational Research).

Kroon, L. G.; Salomon, M.; and Van Wassenhove, L. N. 1993, "Exact and approximation algorithms for the tactical fixed interval scheduling problem," Management Report Series 158, Erasmus University, Rotterdam, The Netherlands.

R. R. Kalmann, Director A310/DC-10/ MD-11 Maintenance, KLM Royal Dutch Airlines, PO Box 7700, 1117 ZL Schiphol Airport, The Netherlands, writes, "I confirm that the system described in the paper "Planning the size and organization of KLM's aircraft maintenance personnel" has proved to be a useful managerial tool for analyzing several strategic and tactical problems that appear in personnel planning for aircraft maintenance." 
Copyright 1994, by INFORMS, all rights reserved. Copyright of Interfaces is the property of INFORMS: Institute for Operations Research and its content may not be copied or emailed to multiple sites or posted to a listserv without the copyright holder's express written permission. However, users may print, download, or email articles for individual use. 